\title{
Effect of delayed sampling on umbilical cord arterial and venous lactate and blood gases in clamped and unclamped vessels
}

\author{
L Armstrong, B Stenson
}

See end of article for authors' affiliations

Correspondence to: Dr Armstrong, Simpson Centre for Reproductive Health, Royal Infirmary of Edinburgh, 51 Little France Crescent, Edinburgh EH16 4SA, Scotland, UK; lazarmstrong@doctors. org.uk

Accepted 8 April 2006 Published Online First 25 April 2006

Background: Cord blood lactate at birth is a marker of antenatal hypoxia, and is comparable to $\mathrm{pH}$ as a prognostic tool.

Objective: To determine, by a prospective observational study, the effect of delayed sampling from arteries and veins that were double clamped to isolate the blood from the placenta (clamped), and from vessels that were not isolated from the placenta (unclamped).

Methods: Paired samples taken from clamped and unclamped vessels at $0,20,40$, and 60 minutes were analysed for lactate, base excess, $\mathrm{pH}$, and $\mathrm{PCO}_{2}$. Data were analysed as the change from time 0 at 20,40, and 60 minutes.

Results: Thirty eight placentas of infants delivered by elective caesarean section were studied. Arterial samples were taken from 20 placentas, and venous samples from 18 placentas. Arterial and venous lactate was significantly higher than at time 0 by 20 minutes in both clamped and unclamped vessels. Changes in unclamped vessels were greater than in clamped vessels. The $\mathrm{pH}$ remained unchanged over 60 minutes in clamped vessels, but changed significantly in unclamped vessels. Base excess changed significantly in both clamped and unclamped vessels.

Conclusions: Cord blood samples taken after 20 minutes delay are unreliable for lactate measurement, even if the vessel has been doubly clamped to isolate the blood from the placenta. Current guidelines that state that blood can be sampled from a clamped cord for up to one hour after delivery should not apply to the interpretation of lactate or base excess. Delayed sampling from unclamped cords is very unreliable.

U mbilical cord blood sampling provides important nformation about an infant's biochemical milieu at the time of delivery. Immediate umbilical cord blood sampling is not always possible, and is often overlooked, especially when a midwife's attention is focused on resuscitation of the infant. When delayed umbilical cord sampling is performed, the results can be difficult to interpret because of the lack of objective data on the reliability of such samples. Current evidence shows that the $\mathrm{pH}, \mathrm{PCO}_{2}$, and $\mathrm{PO}_{2}$ of arterial and venous blood stored in a doubly clamped segment of cord at room temperature can be measured reliably for up to 60 minutes after birth. ${ }^{1-3}$ Professional guidelines recommend circumstances when umbilical cord blood sampling should be undertaken, but do not give clear instructions for sampling methods or guidance on the interpretation of specimens that are obtained after delay from cords that have not been clamped. ${ }^{45}$

Lactate is increasingly recognised as a useful measure of tissue hypoxia in the intensive care setting. Studies in newborn infants have shown that umbilical cord or early life arterial blood lactate correlates well with short and long term neurological outcomes. ${ }^{6-8}$ Although lactate offers an alternative to $\mathrm{pH}$ as a marker of fetal distress, there are no data on how cord arterial and venous lactate measurements may be affected by delayed sampling, or by sampling from a vessel that was not clamped at birth.

We performed a prospective observational study to examine how cord lactate and blood gas measurements are affected by the time period between delivery and sampling. Furthermore, we investigated how these measurements differ when sampled from a doubly clamped umbilical cord (clamped) compared with a vessel that remains in continuity with the placenta (unclamped). The aim of the study was to aid interpretation of cord lactate measurements as well as cord gases when there has been considerable delay in sampling from either a clamped or an unclamped vessel.

\section{METHODS}

We studied placentas of infants undergoing elective caesarean section at full term. In each case, the umbilical cord was doubly clamped by the obstetrician at the time of delivery to isolate arterial and venous blood from the placenta. This segment of cord was then clamped a further three times by the researcher to isolate four individual segments of umbilical cord (fig 1). We obtained a blood sample as soon as possible after birth from one clamped segment, and, immediately afterwards, we sampled from a vessel at the base of the cord that remained in continuity with the placenta (unclamped). In this way, we obtained paired samples from clamped and unclamped vessels. The initial paired samples were obtained within two to three minutes of delivery of the infant, and termed time 0 . Further paired clamped and unclamped samples were obtained at 20, 40, and 60 minutes after delivery. Paired clamped and unclamped samples were always obtained from the same type of vessel, either arterial or venous. Placentas were stored in an open container at room temperature $\left(22-24^{\circ} \mathrm{C}\right)$ during the sampling period. Samples were taken in heparinised syringes and analysed immediately for lactate and blood gases using a Radiometer ABL 700 series.

The primary aim was to characterise the magnitude of change from time 0 in arterial and venous lactate and blood gas measurements in samples taken from clamped and unclamped vessels. The secondary aim was to compare the samples taken from clamped vessels with those from unclamped vessels at the respective time points. 


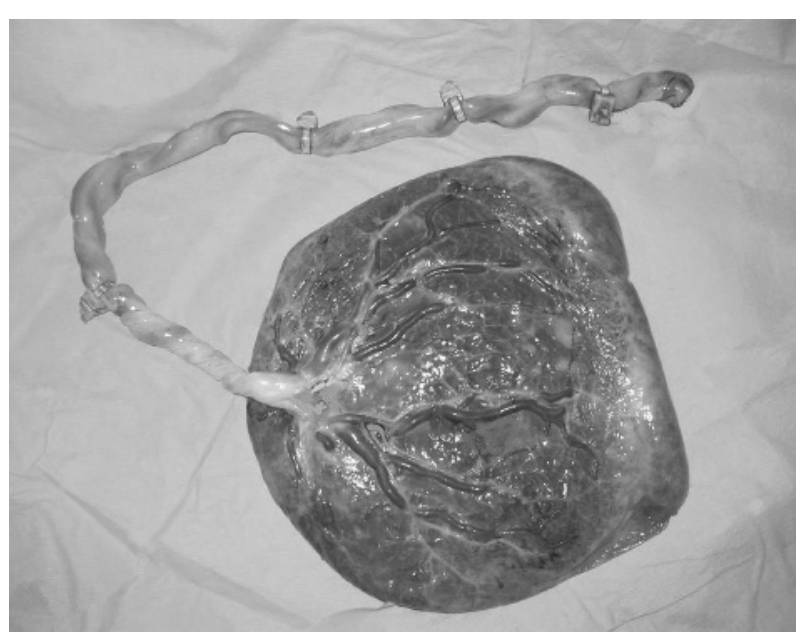

Figure 1 Placenta with umbilical cord clamps in place isolating aliquots of cord blood from the placenta at the time of delivery.

Results were expressed as the change over time in each measured variable from the value at time 0 . Longitudinal data were compared using analysis of variance. Post hoc analyses included the Bonferroni correction for multiple comparisons. Cross sectional comparison between clamped and unclamped data used the paired samples $t$ test. Data were analysed using SPSS version 11.5. Informed written consent for the study was obtained from the mothers undergoing caesarean section. The study was approved by the local ethics advisory committee.

Table 1 Results of arterial and venous samples taken from clamped and unclamped vessels at time 0

\begin{tabular}{|c|c|c|}
\hline & $\begin{array}{l}\text { Arterial } \\
(n=20)\end{array}$ & $\begin{array}{l}\text { Venous } \\
(n=18)\end{array}$ \\
\hline \multicolumn{3}{|c|}{ Lactate (mmol/l) } \\
\hline Clamped & $2.74(1.13)$ & $2.59(1.25)$ \\
\hline Unclamped & $2.69(1.07)$ & 2.51 (1.09) \\
\hline \multicolumn{3}{|c|}{ Base excess (mmol/l) } \\
\hline Clamped & $-1.85(1.38)$ & $-3.08(2.35)$ \\
\hline Unclamped & $-1.54(1.72)$ & $-2.57(2.10)$ \\
\hline \multicolumn{3}{|l|}{$\mathrm{pH}$} \\
\hline Clamped & $7.28(0.05)$ & $7.30(0.05)$ \\
\hline Unclamped & $7.30(0.05)^{*}$ & 7.30 (0.05) \\
\hline \multicolumn{3}{|l|}{$\mathrm{PCO}_{2}(\mathrm{kPa})$} \\
\hline Clamped & $7.03(0.92)$ & $6.24(1.10)$ \\
\hline Unclamped & $6.73(0.89)$ & $6.51(1.17)$ \\
\hline
\end{tabular}

Data are mean (SD)

*Significantly different from clamped value, $\mathrm{p}<0.05$.

\section{RESULTS}

We studied blood samples from 38 placentas, obtaining paired arterial samples from 20 placentas, and paired venous samples from 18 placentas. All placentas were obtained from mothers undergoing elective caesarean section at term under regional anaesthesia.

Table 1 shows the results obtained for arterial and venous samples from clamped and unclamped vessels at time 0 . The mean $\mathrm{pH}$ of unclamped arterial blood was slightly higher than that of clamped blood. Otherwise there were no significant differences between clamped and unclamped samples. All samples had normal values at this time.

Tables 2 and 3 show longitudinal change from time 0 in lactate, base excess, $\mathrm{pH}$, and $\mathrm{PCO}_{2}$ measurements at each time point for clamped and unclamped vessels. Differences that are significant from the value at time 0 are indicated. There was steady accumulation of lactate over time in both arterial and venous blood from both clamped and unclamped vessels. The changes from time 0 were significant by 20 minutes after delivery, and continued to be so at 40 and 60 minutes. Base excess was significantly lower (more negative) than at time 0 from 20 minutes onwards in unclamped arterial blood, and from 40 minutes onwards in all other samples. The $\mathrm{pH}$ of both arterial and venous samples taken from clamped vessels remained unchanged over 60 minutes. However, there was a steady decrease in $\mathrm{pH}$ in samples from unclamped vessels. This had reached a difference of approximately $0.20 \mathrm{pH}$ units in unclamped arterial samples and $0.25 \mathrm{pH}$ units in unclamped venous samples by 60 minutes after delivery. Changes in $\mathrm{PCO}_{2}$ during the sampling period were less consistent. There was a wide scatter of values. At 60 minutes, there was a significant decrease in the $\mathrm{PCO}_{2}$ of arterial blood from clamped vessels, and a significant increase in the $\mathrm{PCO}_{2}$ of venous blood from unclamped vessels.

Figures 2 and 3 compare changes in lactate concentration between clamped and unclamped vessels. Changes in unclamped vessels were significantly greater than in clamped vessels from 20 minutes onwards for both arterial and venous blood.

\section{DISCUSSION}

We found that during the 60 minutes after delivery, there was considerable change in arterial and venous cord blood lactate. Although this change was greater in vessels that remained in continuity with the placenta (unclamped), there was also clinically significant change in blood from vessels that had been doubly clamped to isolate the blood from the placenta. Lactate concentrations in cord blood not sampled immediately after birth should therefore be considered unreliable, whether or not the cord vessels have been clamped. This is in clear contrast with the finding that cord

Table 2 Change from baseline in arterial values at 20,40, and 60 minutes

\begin{tabular}{llll}
\hline & 20 & 40 & 60 \\
\hline Lactate (mmol/I) & $1.2(0.39)^{*}$ & $2.3(0.55)^{*}$ & $3.2(1.23)^{*}$ \\
Clamped & $1.9(0.90)^{*}$ & $5.2(2.04)^{*}$ & $6.6(2.39)^{*}$ \\
Unclamped & $-1.23(1.16)$ & $-2.65(1.56)^{*}$ & $-4.46(2.53)^{*}$ \\
Base excess (mmol/I) & $-2.82(1.89)^{*}$ & $-8.31(3.99)^{*}$ & $-9.20(3.99)^{*}$ \\
Clamped & $-0.011(0.027)$ & $-0.005(0.034)$ & $0.007(0.054)$ \\
Unclamped & $-0.021(0.054)$ & $-0.171(0.142)^{*}$ & $-0.206(0.151)^{*}$ \\
$\mathrm{pH} \quad$ & & $-1.49(2.60)^{*}$ \\
Clamped & $-0.13(0.76)$ & $-0.74(0.75)^{*}$ & $1.46(2.60)$ \\
Unclamped & $-0.42(1.37)$ & $0.95(2.63)$ & \\
$\mathrm{PCO}_{2}$ (mmol/l) & Clamped & &
\end{tabular}

Data are mean (SD)

*Significant deviation from baseline, $\mathrm{p}<0.05$. 


\begin{tabular}{|c|c|c|c|}
\hline & 20 & 40 & 60 \\
\hline \multicolumn{4}{|l|}{ Lactate $(\mathrm{mmol} / \mathrm{l})$} \\
\hline Clamped & $0.9(0.71)^{*}$ & $1.5(0.75)^{*}$ & $2.2(0.76)^{*}$ \\
\hline Unclamped & $1.9(1.71)^{*}$ & $3.4(2.22)^{*}$ & $5.4(2.69)^{*}$ \\
\hline \multicolumn{4}{|c|}{ Base excess $(\mathrm{mmol} / \mathrm{l})$} \\
\hline $\begin{array}{l}\text { Clamped } \\
\text { Unclamped }\end{array}$ & $\begin{array}{l}-1.42(2.73) \\
-1.79(4.22)\end{array}$ & $\begin{array}{l}-1.62(1.86)^{\star} \\
-4.36(3.88)^{\star}\end{array}$ & $\begin{array}{l}-2.36(1.27)^{*} \\
-7.58(4.59)^{*}\end{array}$ \\
\hline \multicolumn{4}{|l|}{$\mathrm{pH}$} \\
\hline $\begin{array}{l}\text { Clamped } \\
\text { Unclamped }\end{array}$ & $\begin{array}{l}-0.014(0.023) \\
-0.077(0.085)\end{array}$ & $\begin{array}{l}-0.013(0.032) \\
-0.134(0.104)^{*}\end{array}$ & $\begin{array}{l}-0.006(0.041) \\
-0.252(0.150)^{*}\end{array}$ \\
\hline \multicolumn{4}{|l|}{$\mathrm{PCO}_{2}(\mathrm{mmol} / \mathrm{l})$} \\
\hline Clamped & $0.08(0.67)$ & $0.18(0.47)^{*}$ & $0.42(0.67)$ \\
\hline Unclamped & $0.94(1.53)$ & $1.61(1.98)$ & $3.67(3.03)^{*}$ \\
\hline
\end{tabular}

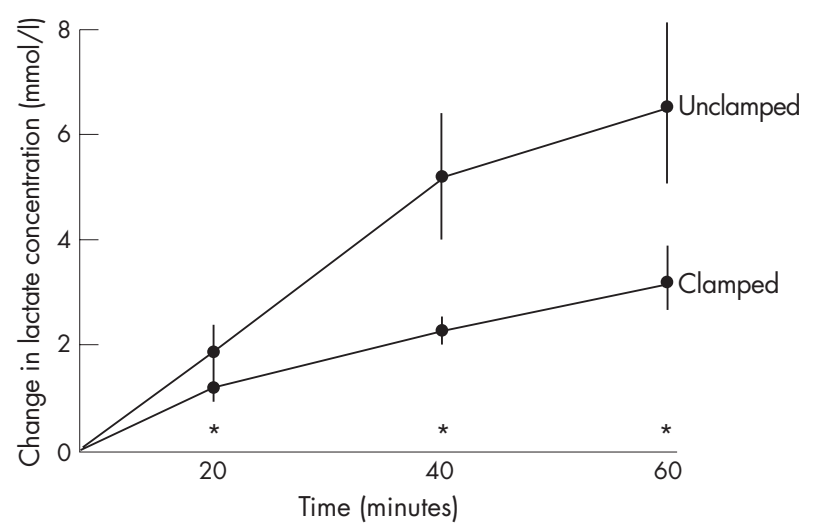

Figure 2 Change in arterial lactate concentration observed in clamped and unclamped vessels. Data are mean (SEM). *Significant difference between magnitude of change in clamped versus unclamped samples at corresponding time point $(p<0.05)$.

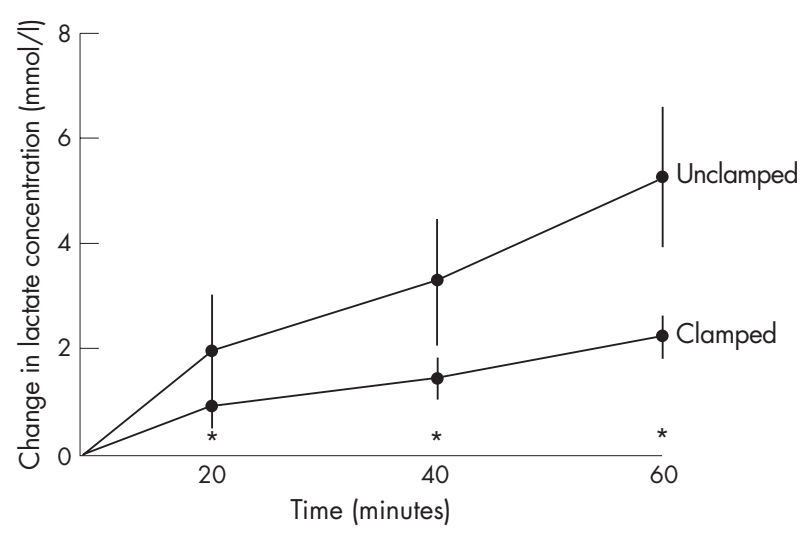

Figure 3 Change in venous lactate concentration observed in clamped and unclamped vessels. Data are mean (SEM). *Significant difference between magnitude of change in clamped versus unclamped samples at corresponding time point $(\mathrm{p}<0.05)$.

arterial and venous $\mathrm{pH}$ measurements remain reliable for an hour if they are made on blood that is sampled from a clamped section of cord..$^{1-3}$

The increasing lactate concentrations reflect continuing anaerobic glycolysis. The rise observed in blood sampled from clamped vessels presumably indicates metabolism in erythrocytes, leucocytes, and endothelial cells. The larger accumulation observed in blood that had remained in continuity with the placenta indicates a much larger metabolic contribution from the placenta. Lactate measurements have been used as an index of illness severity and a predictor of outcome in infants undergoing neonatal intensive care. ${ }^{9-11}$ Piquard and colleagues ${ }^{12}$ showed that umbilical cord blood lactate is predominantly fetal in origin, rather than maternal, and that umbilical cord venous lactate concentrations are not usually higher than umbilical cord arterial lactate concentrations. In circumstances of hypoxic ischaemic encephalopathy, umbilical cord blood and early neonatal arterial blood lactate may be a useful predictor of the severity of neonatal encephalopathy ${ }^{73}$ and longer term neurodevelopmental outcome. ${ }^{68}$

Ackerman and colleagues ${ }^{14}$ were the first to study the effect of delayed sampling on the $\mathrm{pH}$ and $\mathrm{PCO}_{2}$ of cord arterial blood. They showed significant change in both variables within the first minute of birth, indicating the importance of immediate cord clamping. Duerbeck and colleagues ${ }^{1}$ reported that, once cord blood was isolated between clamps, no change occurred in $\mathrm{pH}, \mathrm{PCO}_{2}$, and $\mathrm{Po}_{2}$ over one hour in cords left at room temperature. Similar findings were reported by Sykes et $a l^{2}$ and Hilger et al. ${ }^{3}$ Our findings broadly concur with these data.

There are fewer published data on the effect of delayed sampling on the metabolic status of cord blood. Owen and colleagues $^{15}$ showed no change in the base excess of clamped cord blood stored at room temperature for 30 minutes after delivery. We found that important changes had occurred over 20 and 40 minutes in arterial and venous blood respectively. Sinn and colleagues ${ }^{16}$ showed that lactate concentration of blood contained in a syringe stored at room temperature rises significantly within five minutes of storage. Our study specifically observes the effect of delayed sampling rather than delayed analysis. Interestingly, the magnitude of change in the samples studied by Sinn and colleagues is much smaller than in our study. This suggests that umbilical vessel endothelium contributes significantly to the ongoing metabolism.

This study shows changes in samples from infants who were metabolically normal at delivery. It does not show what may happen in samples that are originally of borderline or abnormal metabolic status. The linearity of the changes in lactate does, however, indicate that lactate production is uniform in both a normal biochemical milieu (time 0 ) and an abnormal one (subsequent times). However, by studying this particular cohort, the study does show that a normal metabolic status rapidly becomes abnormal as a result of artefact.

This study is applicable to a small but high risk cohort of deliveries. Delayed cord sampling may be performed after unexpected emergency during home birth, when infants have been born before arrival in hospital or when emergencies during births in hospital dictate that the available staff have greater clinical priority than immediate cord blood sampling. In each case, the infants may be at higher risk of adverse 


\section{What is already known on this topic}

- Lactate is a useful index of fetal anaerobic metabolism and is as good a prognostic tool as acid-base status

- There is no significant change in $\mathrm{pH}$ and blood gases in arterial blood in a clamped vessel stored at room temperature for up to one hour

\section{What this study adds}

- Measurements of lactate concentration in arterial and venous umbilical cord blood become unreliable within 20 minutes of delivery whether sampled from clamped or unclamped vessels

- Blood sampled from unclamped vessels becomes unreliable for interpretation of blood gases within 20 minutes

outcome, and cord blood analysis may prove to be important both clinically and medicolegally. Professional guidelines recommend that a clamped cord can be left for up to one hour at room temperature before being sampled for blood gas analysis. ${ }^{4}$ This is likely to produce reliable $\mathrm{pH}$ results if the blood is isolated from the placenta, but we show here that lactate and base excess results should not be considered reliable under these circumstances. Future guidance should also stress clearly that delayed sampling of cord blood that has not been isolated from the placenta by a clamp is unreliable for all variables.

\section{Authors' affiliations}

L Armstrong, B Stenson, Simpson Centre for Reproductive Health, Royal Infirmary of Edinburgh, Edinburgh, Scotland, UK
Competing interests: none declared

\section{REFERENCES}

1 Duerbeck NB, Chaffin DG, Seeds JW. A practical approach to umbilical artery $\mathrm{pH}$ and blood gas determinations. Obstet Gynecol 1992;79:959-62.

2 Sykes GS, Molloy PM. Effect of delays in collection or analysis on the results of umbilical cord blood measurements. Br J Obstet Gynaecol 1984;91:989-92.

3 Hilger JS, Holzman IR, Brown DR. Sequential changes in placental blood gases and $\mathrm{pH}$ during the hour following delivery. J Reprod Med 1981;26:305-7.

4 ACOG technical bulletin. Umbilical artery blood acid-base analysis. Int J Gynaecol Obstet 1996;52:305-10.

5 Royal College of Obstetricians and Gynaecologists Clinical Effectiveness Support Unit. The use of electronic fetal monitoring: the use and interpretation of cardiotocography in intrapartum fetal surveillance. London: RCOG, 2001. http://www.rcog.org.uk/resources/public/pdf/

efm_guideline_final_2may2001.pdf (accessed 26 June 2006).

6 Groenendaal F, Lindemans C, Uiterwaal CS, et al. Early arterial lactate and prediction of outcome in preterm neonates admitted to a neonatal intensive care unit. Biol Neonate 2003;83:171-6.

7 da Silva S, Hennebert N, Denis R, et al. Clinical value of a single postnatal lactate measurement after intrapartum asphyxia. Acta Paediatr 2000:89:320-3

8 Westgren $M$, Divon $M$, Horal $M$, et al. Routine measurements of umbilical artery lactate levels in the prediction of perinatal outcome. Am J Obstet Gynecol 1995; 173:1416-22.

9 Cheung PY, Etches PC, Weardon M, et al. Use of plasma lactate to predict early mortality and adverse outcome after neonatal extracorporeal membrane oxygenation: a prospective cohort in early childhood. Crit Care Med 2002;30:2135-9.

10 Cheung PY, Robertson CM, Finer NN. Plasma lactate as a predictor of early childhood neurodevelopmental outcome of neonates with severe hypoxaemia requiring extracorporeal membrane oxygenation. Arch Dis Child Fetal Neonatal Ed 1996;74:F47-50.

11 Deshpande SA, Platt MP. Association between blood lactate and acid-base status and mortality in ventilated babies. Arch Dis Child Fetal Neonatal Ed 1997;76:F15-20.

12 Piquard F, Schaefer A, Dellenbach $P$, et al. Is fetal acidosis in the human fetus maternogenic during labor? A reanalysis. Am J Physiol 1991;261:R1294-9.

13 Chou YH, Tsou YK, Wang PJ. Clinical application of the measurement of cord plasma lactate and pyruvate in the assessment of high-risk neonates. Acta Paediatr 1998;87:764-8.

14 Ackerman BD, Sosna MM, Ullrich JR. A technique for serial sampling of umbilical artery blood at birth. Biol Neonate 1972;20:458-65.

15 Owen P, Farrell TA, Steyn W. Umbilical cord blood gas analysis: a comparison of two simple methods of sample storage. Early Hum Dev 1995;42:67-71.

16 Sinn JK, Lloyd J, Todd DA, et al. Umbilical cord blood lactate in normal infants: comparison between two methods of measurement. J Paediatr Child Health 2001;37:24-7.

\section{Call for abstracts}

International Forum on Quality \& Safety in Health Care

18-20 April 2007, Palau De Congressos, Barcelona

Deadline: 25 September 2006

http://www.quality.bmipg.com 